\title{
Three-dimensional nucleus-to- cytoplasm ratios provide better discrimination of normal and lung adenocarcinoma cells than in two dimensions
}

\author{
Hsu-Cheng Huang \\ Shu-Jen Chiang \\ Shu-Han Wen \\ Pei-Jung Lee \\ Huei-Wen Chen \\ Yang-Fang Chen \\ Chen-Yuan Dong
}


Three-dimensional

nucleus-to-cytoplasm

ratios provide better

discrimination of normal

and lung adenocarcinoma

cells than in two dimensions

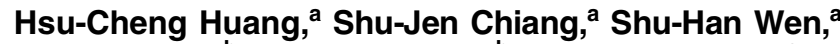 \\ Pei-Jung Lee, ${ }^{b}$ Huei-Wen Chen, ${ }^{b}$ Yang-Fang Chen, ${ }^{a}$ \\ and Chen-Yuan Dong ${ }^{a, *}$ \\ ${ }^{a}$ National Taiwan University, Department of Physics, Taipei, Taiwan \\ ${ }^{b}$ National Taiwan University, College of Medicine, Graduate Institute \\ of Toxicology, Taipei, Taiwan
}

\begin{abstract}
We acquired multiphoton images of normal and lung adenocarcinoma cell lines in three dimensions. Image stacks of the cells were then processed to obtain nucleusto-cytoplasm (N/C) ratios in two and three dimensions. While $\mathrm{N} / \mathrm{C}$ ratios in three dimensions can be unambiguously determined from the volumetric ratios of the nucleus and cytoplasm, two-dimensional (2-D) N/C can vary depending on the axial plane selected for N/C ratio determination. We determined $2-\mathrm{D} N / \mathrm{C}$ ratios from three criteria: (1) axial position at which the nuclear area is the largest; (2) the largest 2-D N/C ratio value; and (3) axial position at the midpoint of nuclear axial position. We found that different definitions of 2-D N/C ratio will significantly affect its value. Furthermore, in general, larger variance was found in 2-D rather than three-dimensional (3-D) N/C ratios. Lack of ambiguity in definition and reduced variance suggest that 3-D N/C ratio is a better parameter for characterizing tumor cells in the clinical setting. $\odot$ The Authors. Published by SPIE under a Creative Commons Attribution 4.0 Unported License. Distribution or reproduction of this work in whole or in part requires full attribution of the original publication, including its DOI. [DOI: 10.1117/1.JBO.24.8.080502]
\end{abstract}

Keywords: optics; two-photon microscopy; lung cancer; nucleus-tocytoplasm -ratio.

Paper 190067LRR received Apr. 1, 2019; accepted for publication Jul. 17, 2019; published online Aug. 20, 2019.

\section{Introduction}

The gold standard of clinical diagnosis of diseases, particularly those in discriminating of normal and cancerous tissues, remains to be histology. In the most common implementation, tissue of interest is fixed, paraffin-imbedded, thinly sliced, and labeled with hematoxylin and eosin for optical microscopic examination. Pathologist then determines the pathological state of the tissue specimens by qualitative examination and

*Address all correspondence to Chen-Yuan Dong, E-mail: cydong@ phys.ntu .edu.tw semiquantitative scoring. In the case of cancer, a positive identification is often attributed to a higher nuclear-to-cytoplasm (N/C) ratio characteristic of tumor cells. Particularly, N/C ratio analysis has been applied to characterize oral smears, ${ }^{1}$ nuclearto-cytoplasmic change is indicative of dysplastic change in oral tissues, ${ }^{2}$ and high CDK1 N/C ratios correlate to poor prognosis in colorectal cancer patents. ${ }^{3}$ Furthermore, two-color, two-photon imaging found larger N/C ratio in colon adenocarcinoma specimens. ${ }^{4}$ In addition to tissue-based histology, cytological procedures, such as Pap smear, and analysis based on bodily fluids, such as sputum, urine, and peritoneal fluid, are heavily used in the clinical setting. Despite the wide use of N/C ratio, significant variations among observers were found in nuclear ratios and interpersonal variations in N/C ratio determination, a persistent problem in histological analysis. ${ }^{5,6}$

Uncertainty of using N/C ratio for disease diagnosis is due to the fact that traditionally, N/C ratio analysis is two-dimensional (2-D) in nature. Since the tissue slices are on the order of a few microns, diagnosis of such specimens is a 2-D representation of the actual specimen. Depending on the physical location and orientation of the tissue section, simulations have shown that geometric factors, such as nuclear position, cell shape, and orientation, can significantly affect $2-\mathrm{D}$ N/C ratios. ${ }^{7}$ Therefore, a more precise definition of N/C ratio would result in reduced ambiguity in clinical differentiation of tumor from normal cells. While techniques such as automatic digital extraction of morphological features, three-dimensional (3-D) reconstruction of serial histological images, and 3-D N/C ratio obtained from optical coherence tomography have been developed for improved cancer identification, ${ }^{8-10}$ a comparison of the efficacy between 2-D and 3-D N/C ratio analysis through dual-color fluorescence analysis is absent. In this study, we imaged both normal and lung adenocarcinoma cells by 3-D multiphoton microscopy. By labeling the nuclei and cytoplasm with fluorescent dyes of different spectral properties, we acquired serial images in three dimensions for determination of 2-D and 3-D N/C ratios.

\section{Materials and Methods}

\subsection{Multiphoton Microscope}

The multiphoton microscope used in this study is a home-built, inverted system. The 780-nm output of a diode-pumped solidstate laser (Millennia, Spectra Physics, Santa Clara, California) titanium-sapphire laser (Tsunami, Spectra Physics) was used as the excitation source. Upon reflecting from a galvanometerdriven $x-y$ mirror system, the laser is guided into an inverted microscope (TE2000U, Nikon, Japan). A pair of beam expanders at the input of the microscope is used to enlarge the laser spot in order to ensure overfilling of the focusing objective. The expanded beam is then reflected into the focusing objective by a primary dichroic mirror (720DCLR, Chroma Technology, Bellows Falls, Vermont). The objective used was an air lens (Nikon S Fluor $20 \times /$ NA 0.75) with a working distance of $1 \mathrm{~mm}$, and the on-sample power was $50 \mathrm{~mW}$. The signal generated at the focal volume is collected by the focusing objective in the epi-illuminated geometry and separated into a generic four-channel photomultiplier detection system. The use of additional dichroic mirrors (550DCXR, 495DCXR, and 420DCLR) and bandpass filters (red: $610 / 75 \mathrm{~nm}$, green: $525 / 50 \mathrm{~nm}$, blue: $460 / 50 \mathrm{~nm}$, and second-harmonic generation: 390/18 nm) allow the separation of the signal into three fluorescence and one backward second-harmonic generation channels. In this study, we 
only used the blue and red channels for the detection of Hoechst 33342 labeled nuclei and CellTracker ${ }^{\mathrm{TM}}$ Orange CMTMR labeled cytoplasm, respectively. Each optical image plane is $253 \times 253 \mu \mathrm{m}^{2}$ in size. To achieve a 3-D image, the specimens are placed on a three-axis specimen translation stage (ProScan ${ }^{\circledR}$, Prior Scientific, United Kingdom). After an optical image is acquired in the image plane, the specimen is translated to the next axial position for further image acquisition. In this manner, we acquired 3-D images separated by $1 \mu \mathrm{m}$. A 3-D image stack is typically composed of 20 to 30 images.

\subsection{Preparation of Normal and Lung Adenocarcinoma Cell Lines}

To compare 2-D and 3-D N/C ratios, we used one normal lung epithelial line (Beas2B) and two lung adenocarcinoma cell lines (CL1-0 and CL1-5). ${ }^{11}$ The cell lines were cultured in Roswell Park Memorial Institute 1640 (89\%) supplemented with 10\% fetal bovine serum and $1 \%$ Pen Strep $(10,000$ units $/ \mathrm{mL} /$ penicillin $10,000 \mu \mathrm{g} / \mathrm{mL}$ ) under $37^{\circ} \mathrm{C}$ and $5 \% \mathrm{CO}_{2}$. Upon reaching $90 \%$ confluency, the cells were detached from the culturing flasks with trypsin $0.05 \%$ and ethylenediaminetetraacetic acid and scraping. At this point, the cells can be cultured in No. 1.5 cover glass bottom chambers between 16 and $24 \mathrm{~h}$, prior to labeling. To label the cytoplasm, $20 \mu \mathrm{M}$ in phosphate-buffered saline (PBS) buffer of CellTracker ${ }^{\mathrm{TM}}$ Orange CMTMR (Invitrogen, Grand Island, New York) was first applied for 30 min. Following three times washing with PBS, the cells are labeled with Hoechst 33342 (Invitrogen) at the concentration of $0.4 \mu \mathrm{g} / \mathrm{mL}$ for $10 \mathrm{~min}$. The cells were then again washed three times with PBS buffer before mounting on the multiphoton microscope for imaging.

For each cell line, high-resolution, dual-color multiphoton imaging of 20 cells in three dimensions were acquired. Following image acquisition, 2-D images at different axial positions are analyzed for N/C ration analysis. For this purpose, background noise was determined from regions of the blue and red channel images without cells. These background levels were used as threshold values for the respective blue and red fluorescence images. For determination of the cytoplasmic area, the image of the nucleus was used as mask. The images were then processed by determining the number of pixels from the images of each fluorescence channel. In this manner, the nuclear $(\mathrm{N})$ and cytoplasmic (C) areas of each 2-D image are determined. These values were then used to determine the 2-D N/C ratio each focal plane.

\section{Results}

Figure 1(a) shows the representative multiphoton images of the Beas2B, CL1-0, and CL1-5 cells in three dimensions. Individual 2-D images at different focal depths can then be extracted [Fig. 1(b)]. Qualitatively, the nucleus is largest for CL1-5, followed by CL1-0, and Beas2B. The total size of the cells also follows the same trend. Furthermore, 2-D N/C ratio at each depth was determined. The results show the uncertainty in the precise determination of $2-\mathrm{D}$ N/C ratio value. Specifically, images for all three cells at the depths of 10 and $15 \mu \mathrm{m}$ are similar in appearance; however, the 2-D N/C ratios can differ from 0.36 to 0.26 (Beas2B), 0.54 to 0.42 (CL1-0), and 0.96 to 0.72 (CL1-5).

The ambiguity that exists in a precise definition of N/C ratio in two dimensions is further demonstrated in Fig. 2 where we plotted the nuclear area, cytoplasmic area, 2-D N/C ratio, and total cell area as a function of axial position. In the three representative cells, the axial location of maximum nuclear size and 2-D NC ratio do not correspond and can differ in the 4 to $8 \mu \mathrm{m}$ range. Furthermore, depending on the axial position chosen to calculate the 2-D N/C ratio, the values can differ by a factor of 2 .

To quantify different values of N/C ratio in two dimensions, we applied three definitions of 2-D N/C ratios: (1) $N_{\max }$ : largest nuclear area. N/C ratio was calculated at an axial position at which the nuclear area is the largest; (2) $N / C_{\max }$ : after determining the $\mathrm{N} / \mathrm{C}$ ratio at different depths, the largest $\mathrm{N} / \mathrm{C}$ ratio value was selected; and (3) $N_{\text {midpoint }}$ : midpoint of nuclear axial position was determined and N/C ratio calculated. In addition, the nuclear and cytoplasmic volumes were calculated by summing
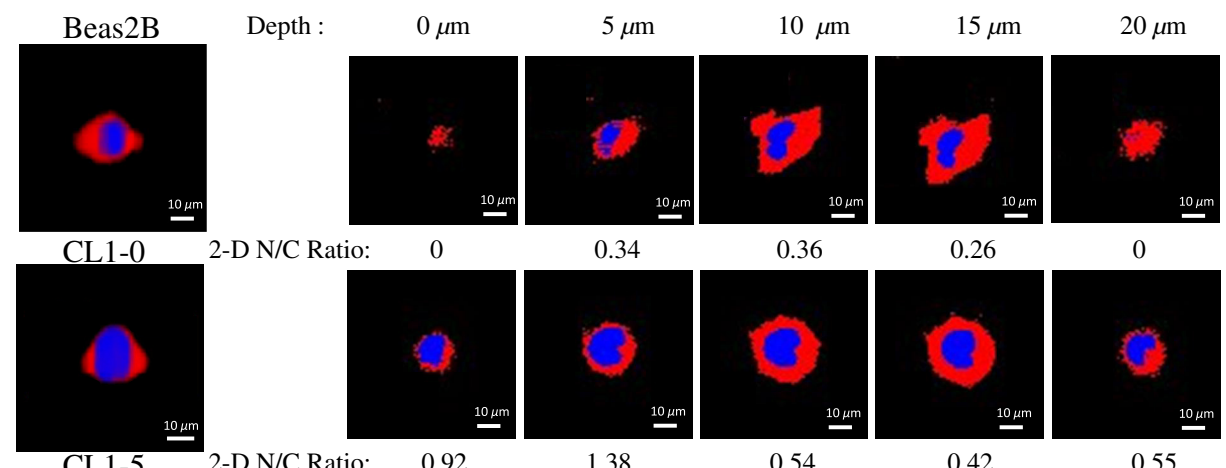

2-D N/C Ratio:
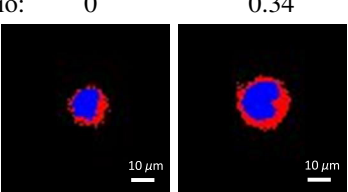

0.36

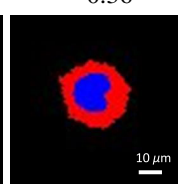

0.26

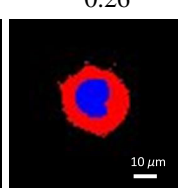

0

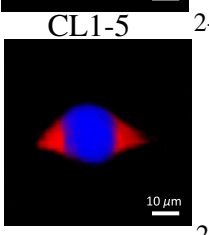

-D N/C Ratio:

1.38

0.54
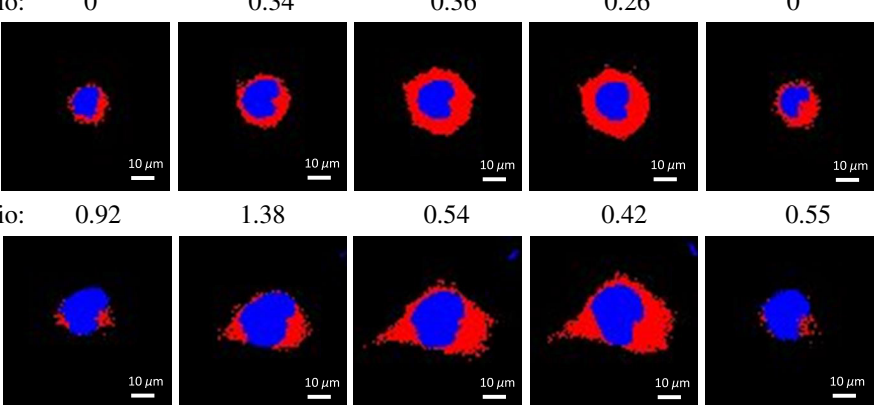

2.2

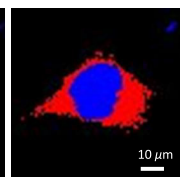

(b)

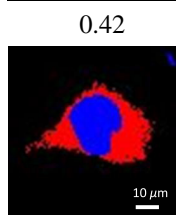

0.72

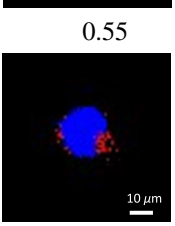

4.8

Fig. 1 (a) Representative multiphoton images of the Beas2B, CL1-0, and CL1-5 cells in three dimensions. (b) The 2-D images of the three cells along with 2-D N/C ratio at different focal depths. Blue pseudocolor: Hoechst 33342 labeled nucleus; red pseudocolor: CellTracker ${ }^{\mathrm{TM}}$ Orange CMTMR labeled cytoplasm. 


\section{JBO Letters}

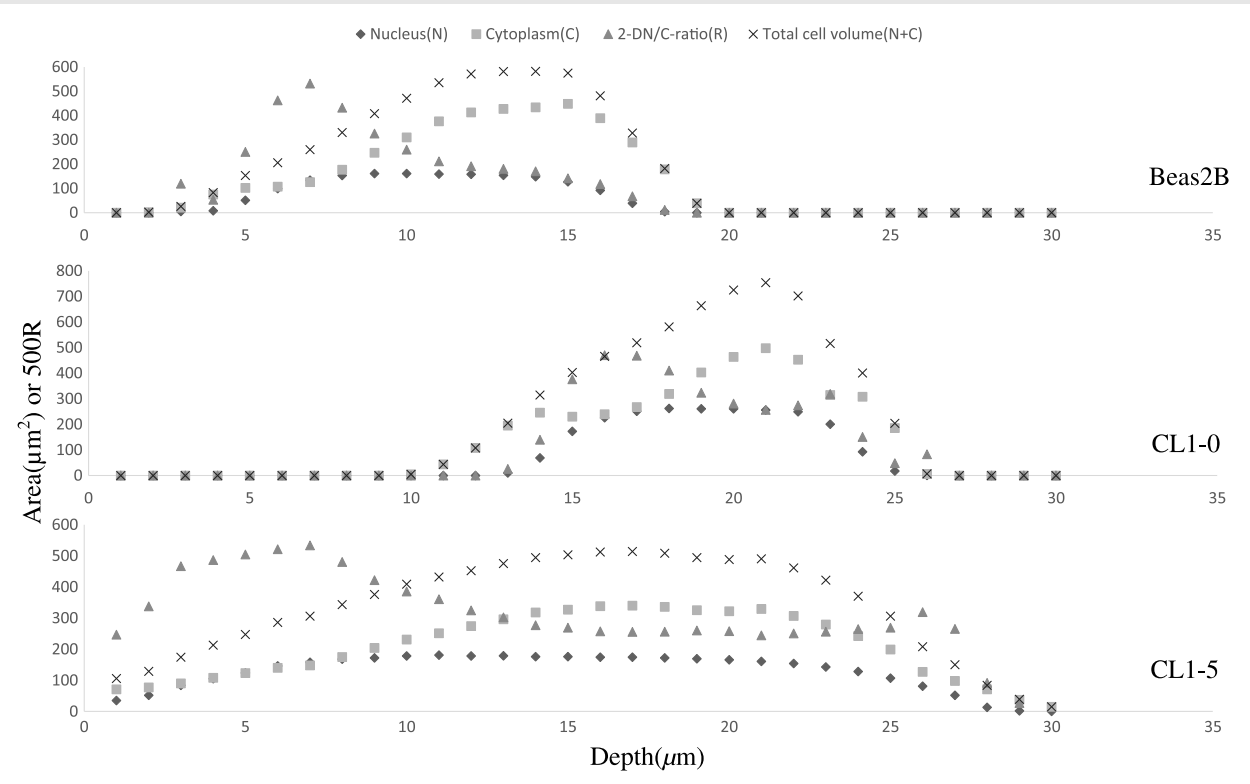

Fig. 2 Representative axial profiles of nuclear area $(N)$, cytoplasmic area $(C)$, total cell area $(N+C)$, and 2-D N/C ratio (500X) for Beas2B, CL1-0, and CL1-5 cell lines.

Table 1 Statistical analysis of the physical characteristics of Beas2B, CL1-0, and CL1-5 cell lines in two and three dimensions (20 cells in each cell line were analyzed).

\begin{tabular}{|c|c|c|c|c|c|c|c|}
\hline & \multirow{2}{*}{$\begin{array}{c}\text { Nucleus } \\
\text { volume }\left(\mu \mathrm{m}^{3}\right)\end{array}$} & \multirow{2}{*}{$\begin{array}{c}\text { Cytoplasm } \\
\text { volume }\left(\mu \mathrm{m}^{3}\right)\end{array}$} & \multirow{2}{*}{$\begin{array}{c}\text { Total cell volume } \\
\text { (nucleus }+ \text { cytoplasm) } \\
\left(\mu \mathrm{m}^{3}\right)\end{array}$} & \multirow[b]{2}{*}{ 3-D N/C ratio } & \multicolumn{3}{|c|}{ 2-D N/C ratio } \\
\hline & & & & & $N_{\max }$ & $N / C_{\max }$ & $N_{\text {midpoint }}$ \\
\hline Beas2B & $1787 \pm 584$ & $4756 \pm 1826$ & $6543 \pm 1917$ & $0.40 \pm 0.14(38 \%)$ & $0.59 \pm 0.29(49 \%)$ & $1.07 \pm 0.85(79 \%)$ & $0.46 \pm 0.19(41 \%)$ \\
\hline CL1-0 & $2758 \pm 792$ & $3800 \pm 1038$ & $6558 \pm 1306$ & $0.75 \pm 0.19(28 \%)$ & $0.94 \pm 0.3(33 \%)$ & $3.38 \pm 2.92(86 \%)$ & $0.76 \pm 0.16(21 \%)$ \\
\hline CL1-5 & $3271 \pm 1226$ & $5239 \pm 1896$ & $8510 \pm 2258$ & $0.67 \pm 0.21(31 \%)$ & $0.75 \pm 030(40 \%)$ & $2.28 \pm 1.82(80 \%)$ & $0.62 \pm 0.26(41 \%)$ \\
\hline
\end{tabular}

over the nuclear and cytoplasmic areas at each axial position. Finally, by summing nuclear and cytoplasmic areas at each focal plane, 3-D N/C ratio can be determined. In addition to showing the average value and standard deviations of these parameters, we also determined the percentage of data deviating from the average values (Table 1).

\section{Discussion}

In this study, we studied 2-D N/C ratios determined from different criteria and 3-D volumetric N/C ratio for normal lung epithelial cell line Beas2B and two lung adenocarcinoma cell lines (CL1-0 and CL1-5) cell lines. We found that depending on the criterion chosen, 2-D N/C ratios can vary drastically (Table 1). Particularly, the variance of the data accumulated from 20 cells varies significantly ( $21 \%$ to $86 \%$ ), suggesting that $2-\mathrm{D}$ N/C ratio is critically affected by an individual cell's geometrical orientation at the growth site. On the other hand, the 3-D volumetric N/ $\mathrm{C}$ ratio is unambiguous in definition and shows a lower variance ( $28 \%$ to $38 \%$ ). In addition to higher 3-D N/C ratios for cancer cells, we found that the nuclear volumes for CL1-0 $\left(2768 \mu \mathrm{m}^{3}\right)$ and CL1-5 $\left(3271 \mu \mathrm{m}^{3}\right)$ are significantly larger than that of Beas2B $\left(1787 \mu \mathrm{m}^{3}\right)$ where the cytoplasmic volume of CL1-5 $\left(5239 \mu \mathrm{m}^{3}\right)$ is the largest, followed by Beas $2 \mathrm{~B}\left(4756 \mu \mathrm{m}^{3}\right)$, and CL1-0 $\left(3800 \mu \mathrm{m}^{3}\right)$. Therefore, the more metastatic CL1-5 ${ }^{11}$ is the largest cell type in nucleus and cytoplasm, although it has a smaller 3-D N/C ratio than CL1-0. Since the 3-D N/C ratio for Beas2B and CL1-5 statistically overlap, our results suggest that in addition to $\mathrm{N} / \mathrm{C}$ ratio analysis in three dimensions, other metrics such as nuclear, cytoplasmic, and total cell size should be included for distinguishing between normal and cancerous cells in the clinical setting. If spatial resolution is defined as the size of the focal spot defined by first minima of the Airy pattern, then our use of an NA 0.75 objective, at $780 \mathrm{~nm}$, would result in nominal lateral and axial resolutions are 1.27 and $5.55 \mu \mathrm{m}$, respectively. Since images were acquired at different depths, the use of objectives with different NAs would affect the N/C ratio results. In the case of low NA, although a larger axial depth is sampled, the corresponding decrease in lateral resolution may introduce ambiguity in lateral determination of the size of cellular components. The approach described in this work may be applied to pathological analysis in cytology.

\section{Disclosures}

The authors have no relevant financial interests in this letter.

\section{Acknowledgments}

This work was supported by the Ministry of Science and Technology (MOST 107-2112-M-002-023-MY3). 


\section{JBO Letters}

\section{References}

1. J. G. Cowpe, R. B. Longmore, and M. W. Green, "Quantitative exfoliative cytology of normal oral squames: an age, site and sex-related survey," J. R. Soc. Med. 78(12), 995-1004 (1985).

2. V. Hegde, "Cytomorphometric analysis of squames from oral premalignant and malignant lesions," J. Clin. Exp. Dent. 3(5), e441-e444 (2011).

3. W. W. Sung et al., "High nuclear/cytoplasmic ratio of Cdk1 expression predicts poor prognosis in colorectal cancer patients," BMC Cancer 14, 951 (2014).

4. C. Su Lim et al., "Measurement of the nucleus area and nucleus/cytoplasm and mitochondria/nucleus ratios in human colon tissues by dualcolour two-photon microscopy imaging," Sci. Rep. 5, 18521 (2015).

5. J. L. Schmidt et al., "Visual estimates of nucleus-to-nucleus ratios. Can we trust our eyes to use the Bethesda ASCUS and LSIL size criteria?" Cancer 114, 287-293 (2008).

6. L. J. Vaickus and R. H. Tambouret, "Young investigator challenge: the accuracy of the nuclear-to-cytoplasmic ratio estimation among trained morphologists," Cancer Cytopathol. 123(9), 524-530 (2015).
7. A. G. Nikonenko, "The cell sectioning model. Nuclear/cytoplasmic ratio studied by computer simulation," Anal. Quant. Cytol. Histol. 18(1), 23-34 (1996).

8. M. L. Onozato et al., "A role of three-dimensional (3D)-reconstruction in the classification of lung adenocarcinoma," Anal. Cell. Pathol. (Amst.) 35(2), 79-84 (2012)

9. J. P. Thiran and B. Macq, "Morphological feature extraction for the classification of digital images of cancerous tissues," IEEE Trans. Biomed. Eng. 43(10), 1011-1020 (1996).

10. C. K. Chang et al., "Segmentation of nucleus and cytoplasm of a single cell in three-dimensional tomogram using optical coherence tomography," J. Biomed. Opt. 22(3), 036003 (2017).

11. Y. W. Chu et al., "Selection of invasive and metastatic subpopulations from a human lung adenocarcinoma cell line," Am. J. Respir. Cell Mol. Biol. 17(3), 353-360 (1997). 